\title{
EN TORNO A LAS EXEQUIAS DE FELIPE IV EN SEVILLA
}

\author{
POR JosÉ MANUEl BAENA GALlÉ
}

Las arquitecturas efímeras, que tanta importancia tuvieron en la Edad Moderna, son uno de los aspectos de la historia del arte que mayor interés ha despertado en los últimos años. En estas construcciones, entre las que ocupan un lugar prioritario las de carácter funerario, las instituciones y artistas plasmaron su peculiar visión del mundo y sus gustos estéticos, a la vez que intentaron dejar memoria histórica de los acontecimientos. En este sentido, no se puede olvidar que las arquitecturas efímeras eran un elemento más de un acto cultural complejo donde, la fiesta, la literatura, la música, o el protocolo, entre otros, fueron los ingredientes de actuaciones publicas de gran relevancia. Por ello, es necesario profundizar en el estudio de los túmulos funerarios erigidos en Sevilla como fiel exponente del arte y la mentalidad que caracterizaron cada momento en la vida de la ciudad. Con tal finalidad, se aportan ahora nuevos datos sobre las exequias sevillanas de Felipe IV.

El jueves 17 de septiembre de 1665 moría en Madrid el rey Felipe IV. Este suceso originó la celebración en Sevilla de las ceremonias fúnebres habituales para tales ocasiones, ya estudiadas con anterioridad ${ }^{1}$. Diversas cuestiones, principalmente la falta de medios económicos para costear los actos, hicieron que hasta marzo de 1666 no se pudiesen celebrar los funerales, siendo este retraso una prueba más de la crisis económica en la que se encontraba sumida la ciudad de Sevilla. En estas exequias existió una gran confusión en lo referente a la construcción del túmulo, elemento principal en toda celebración de este tipo. En un primer momento, el Ayuntamiento inició las gestiones para erigir el catafalco funerario, conforme a uno de los dos diseños presentados por Pedro Sánchez Falconete, maestro mayor del municipio. Posteriormente se acordó hacer una especie de concurso publico para

1. Véase, BAENA GALLÉ, José Manuel: Exequias reales en la Catedral de Sevilla durante el siglo XVII. Sevilla, 1992; y "Dibujos arquitectónicos del siglo XVII. Una propuesta de atribución" en Archivo Hispalense, $\mathrm{n}^{\circ} 222$, Sevilla, 1990. Pp. 185-189. 
que los capitulares conociesen otros proyectos y pudiesen elegir con mayor base. La traza que mas gusto al cabildo municipal fue la de Diego de Zúñiga ya que era la "mas autorizada y ... demenos gasto" 2. Tales consideraciones, unidas al hecho de presentar dicho proyecto la planta más "nueva Ylamas dezente yproporcionda" hizo que fuese definitivamente elegida ${ }^{3}$. A la vez, el cabildo nombró los diputados encargados de realizar las gestiones para la construcción del túmulo, quienes debían estar asesorados en estas cuestiones por Sánchez Falconete o Pedro del Valle. Poco más se conoce sobre este túmulo, salvo que fue rematado en Pedro Honorio de Palencia por 43.000 reales de vellón, y que finalmente no fue erigido por problemas de índole económica, aunque sí se inició su construcción, ya que a Palencia se le dieron 400 reales por los gastos que había realizado en la escritura, remate, viajes en busca de la madera, etc. ${ }^{4}$. Por último, se sabe que las condiciones para el referido remate del túmulo las redactó el arquitecto del municipio Pedro Sánchez Falconete. No existían más datos sobre este malogrado proyecto que finalmente fue sustituido por un pequeño túmulo realizado por medio de diversas piezas del ajuar de la Catedral de Sevilla, estructurado gracias a varias plataformas superpuestas y del que se conserva un sencillo dibujo en el archivo del citado templo hispalense.

Por fortuna, recientemente se ha localizado en el Archivo Municipal de Sevilla nueva documentación que permite ampliar los conocimientos sobre este proceso 5 . Gracias a ella es posible una aproximación más exacta sobre cómo se pensaba realizar aquel proyecto y cuáles eran las líneas generales de su composición. Cronológicamente el primer documento corresponde a la memoria y pliego de condiciones relativos a la obra de pintura que se encuentra firmado por Pedro Sánchez Falconete el 31 de octubre de 1665. Mediante aquellas se obligaba a los pintores a poner todo el lienzo necesario, pintar las figuras señaladas y escribir los jeroglíficos habituales en este tipo de edificios. Se debía iniciar el trabajo en el interior de la Lonja, continuando la obra en el espacio de entre coros de la Catedral. El segundo documento es la memoria y condiciones para los carpinteros, que también firma Sánchez Falconete el 1 de noviembre de 1665. En ellas se les obliga a seguir la traza aprobada y a comenzar la construcción en la Lonja, edificio que habitualmente era utilizado por la Catedral para los trabajos iniciales de los montajes fúnebres.

Siguiendo lo expuesto en estos documentos podemos pensar que el túmulo proyectado se levantaba sobre una plataforma unida al ultimo escalón de la capilla

2. Archivo Municipal de Sevilla (A.M.S.). Actas Capitulares de 1665. In Escribanía. Cabildo de viernes 23 de octubre, s.f.

3. A.M.S. Actas Capitulares de 1665. Il Escribanía. Cabildo de viernes 30 de octubre, s.f.

4. Aunque el pintor solicitó 600 reales. Cfr.: A.M.S. Sección $2^{a}$. Archivo de Contaduría. Carpeta 29/1666/doc.95, s.f. y A.M.S. Sección 2. Archivo de Contaduría. Carpeta 123. Data de ordinario y extraordinario 1666.

5. Entre otros documentos se encuentran allí los pliegos de condiciones para carpinteros y pintores redactados por Sánchez Falconete, el acta pública del remate y alguna postura mas de otros artistas sevillanos. A.M.S. Sección $5^{a}$. Escribanías del siglo XVIII. Tomo 188. Doc. $\mathrm{n}^{\circ} 2$. 
mayor de la catedral. El primer cuerpo se formaría por medio de cuatro arcos apoyados sobre pilares con relieves, teniendo en sus claves exteriores las armas de Castilla y en tarjas pequeñas las de Sevilla. En las enjutas de los arcos se pintarian figuras de palomas de la paz con ramos de olivo en el pico. Este cuerpo culminaría en una cornisa con sus resaltes y adornos, sobre la que correría una baranda con cañones para las velas y la iluminación. El resto de la decoración iría pintada imitando la piedra de color pardo. Se rodearía este piso bajo por medio de barandas con cañones de iluminación salvo en las zonas frente a los arcos, para así permitir el acceso a los oficiantes de las ceremonias litúrgicas. El interior se cubriría por medio de una bóveda decorada con castillos y leones -símbolos del reino- y grutescos "contodo lusimiento", siguiendo el gusto del momento.

El segundo cuerpo también se organizaría por medio de arcos -posiblemente de medio punto-, empleándose pintura sobre lienzo para dar forma a las diversas pilastras, dinteles y arquitrabes. En este caso, las superficies irían pintadas imitando el bronce. Aquí, también se utilizaría una cornisa con resaltes y adornos y barandas con cañones para las luces. Este cuerpo se cubriría con una bóveda de media naranja y en su interior se levantaría una tarima cuadrada, de tres baras de lado, y compuesta por tres escalones, sobre la que iría la urna que, con toda seguridad, llevaría decóración de jeroglíficos y motivos heráldicos, y culminaría en las dos almohadas y los atributos de poder real, es decir, corona y cetro. La urna era el elemento central del túmulo y donde en esencia, se encontraba la figura del rey, por lo que se le debía el mayor respeto. Por esa misma razón, ofrecía una mayor riqueza y ornamentación, centrándose en ella la explicación del complejo programa iconográfico.

Por último, el tercer cuerpo se formaría por medio de una plataforma cuadrada sobre la que iría un obelisco en forma de pirámide que se coronaría con una esfera en la que se situaba la figura de la Fama. Esta debía aparecer vestida de blanco y con cota carmesí, llevando alas coloreadas, morión y un clarín "matisado". También en este lugar se colocarían bastidores con velas ${ }^{6}$. Los pintores debían hacer cuatro banderolas de holandilla amarilla con leones y castillos que irían colocadas en las esquinas de la última cornisa del túmulo, siendo su caída cercana a los dos metros.

Para seguridad del edificio se colocarían cuatro cuerdas de cáñamo pintadas que irían amarradas a las partes más firmes de la última cornisa y se atarían a las barandas de las tribunas del templo, dos hacia el coro y dos hacia la capilla mayor. De esta manera se evitaba que el túmulo perdiese su forma por el peso y la altura, o incluso se cayese con los consiguientes efectos desastrosos.

El último aspecto a señalar es que, en las condiciones de construcción, se establecía que tanto los carpinteros como los pintores debían efectuar el trabajo en

6. Todos los cañones para las velas e iluminación del túmulo serían de hojalata "los gruesos de quarta de peralte todos consus orejas para clabadas sobre la madera y los delgados en la mesma conformidad = pidiendo el grueso de la sera todos delgados y gruesos cortados ael talle donde ande serbir para estar a plomo". A.M.S. Sección V. Escribanías del siglo XVIII. Tomo 188. Doc. n² 2. 
mes y medio, empleando para ello todo el personal necesario. Respecto al pago se indica que cobrarían la mitad del dinero durante la obra y el resto al finalizarla, comprometiéndose a desbaratar con toda rapidez el túmulo y a reparar posibles daños en la solería de la catedral. Aunque sería de interés conocer la personalidad de estos artesanos, por el momento es un dato que aún permanece oculto.

Por último, es oportuno señalar que aunque ya era conocido el remate de la obra por 43.000 reales en Pedro Honorio de Palencia, maestro pintor de Sevilla y vecino de la Magdalena, hoy se sabe que la escritura pública correspondiente se firmó el 4 de noviembre de 1665 , aceptándose en la misma las condiciones ya comentadas. En aquel momento fue la oferta más baja, si bien más tarde Alonso Castillejo planteó al Ayuntamiento llevar a cabo las obras por tan solo 39.000 reales de vellón.

Básicamente la idea constructiva de este túmulo del que desgraciadamente -como en tantas otras obras del barroco efímero sevillano- se carece del dibujo correspondiente, parece repetir el esquema seguido en todos los túmulos sevillanos de la primera parte del siglo XVII. Debió poseer planta cuadrada, como era habitual, y un desarrollo vertical por medio de cuerpos superpuestos, que probablemente alcanzase la bóveda del cimborrio de la catedral, conforme a la fórmula de los túmulos-torre, típicos del arte efímero español y según la tradición iniciada en el siglo XVI. En principio, es interesante destacar su parecido con los túmulos de Felipe III, del año 1621, e Isabel de Borbón, de 1644. En todos ellos existía un primer cuerpo de planta cuadrada formado por medio de cuatro arcos de medio punto y un segundo cuerpo, al menos en el proyecto inicial, donde solía radicar la urna. El remate por medio de obelisco o pirámide con una esfera coronándolo era también habitual. Es interesante el hecho de la culminación de la estructura por medio de una figura de gran tamaño, en este caso la Fama, una de las escasas referencias iconográficas del frustrado proyecto de túmulo para Felipe IV en Sevilla, junto con las palomas de la paz y los motivos heráldicos. Dicha figura pretendía incidir en el aspecto más relevante de la persona del monarca, su muerte transcendida, por medio de un concepto tan humanista como es el de la fama, para vivir a través del tiempo y permanecer en la memoria de sus súbditos y en la historia ${ }^{7}$. Más allá de este detalle, es evidente, como se ha señalado, la persistencia de unos modelos estructurales que, se van repitiendo, aunque adecuándose a los gustos estéticos de cada época mediante el uso del lenguaje arquitectónico propio del momento de la construcción. De hecho, pervive la estructura clásica constructiva iniciada en el siglo XVI con el túmulo por la emperatriz Isabel y que solo en 1671 empieza a variar con el monumento por las fiestas de canonización de San Fernando, cambio que tiene su continuidad funeraria en el túmulo de la reina María Luisa de Orleans en 1689, el cual anuncia las variaciones de este tipo de construcciones durante el barroco dieciochesco.

7. En este sentido baste recordar el túmulo de Margarita de Austria en 1611 que coronaba con una escultura del Angel Custodio, del que la reina era muy devota; o el de Felipe III en 1621 que muestra una corona sobre una esfera, simbolizando el triunfo de la monarquía española. 
Por otra parte es notorio el carácter teatral que poseían. estos túmulos. La construcción por medio de lienzos y telas de estos aparatos fúnebres favorecían su altura y ligereza, permitiendo además su rápida ejecución. El público podía observar así un espacio escénico donde el elemento central era el túmulo pero que se encontraba inmerso en todo un conjunto de actuaciones -plásticas, musicales, ceremoniales, etc.- que daban todo su sentido a los actos. Este carácter de telón o decorado, llevaba consigo unos peligros como eran la propia inestabilidad de la maquina -por eso la importancia de arriostrar el túmulo- $\mathrm{y}$, sobre todo, debido a los materiales usados, el temor a un incendio que destruyese todo el trabajo y que afectase incluso al templo y las obras de arte en él conservadas. Otro punto a señalar en estas arquitecturas efímeras, es la importancia otorgada al color. Éste, además de su carácter decorativo posee un marcado carácter simbólico al situarse en los elementos centrales de la escena, tales como la urna o los motivos heráldicos. El color acompaña a toda a toda la construcción, no siendo edificios fríos o monocromos, sino que cada una de sus partes lleva una entonación cromática, lográndose así una gran riqueza visual a la vez que se expresaba con ello unos contenidos simbólicos, típicos de la complejidad cultural del barroco.

Finalmente, es necesario reseñar la responsabilidad de los maestros mayores del Ayuntamiento en la realización de estos aparatos. Ya Soto Caba ha señalado que generalmente la construcción e incluso la traza de los túmulos reales eran monopolio, casi exclusivo, del maestro mayor ${ }^{8}$. En el caso sevillano esto se ve confirmado por las noticias que ahora se dan a conocer. Probablemente el hecho de encargarse de ello los arquitectos municipales es uno de los factores que explican la repetición de modelos, pues en muchas ocasiones se veían condicionados por los gustos oficiales y, lo que es más importante, por las limitaciones presupuestarias. En este sentido es importante señalar el destacado papel de Pedro Sánchez Falconete en las arquitecturas efímeras sevillanas, abarcando su trabajo desde el diseño, caso del túmulo de Isabel de Borbón, en 1644, a la vigilancia del proceso constructivo, como en este de 1665 . Su experiencia en este tipo de actuaciones era ya conocida, siendo preciso insistir en la transcendencia que sus intervenciones tuvieron en el desarrollo de la arquitectura sevillana del seiscientos ${ }^{9}$.

8. SOTO CABA, Victoria: Catafalcos reales del Barroco Español. Un estudio de arquitectura efimera. Madrid, 1991. Pp. 163-172.

9. Véase, CRUZ ISIDORO, Fernando: El Arquitecto Sevillano Pedro Sánchez Falconete. Sevilla, 1991. Asimismo, MORALES, Alfredo J.: "Sobre Pedro Sánchez Falconete, maestro mayor del Ayuntamiento de Sevilla" en Archivo Hispalense, n²229, Sevilla, 1992. Pp. 131-151. 


\section{APÉNDICE DOCUMENTAL}

\section{A.M.S. Sección V. Escribanías del siglo XVIII. Tomo 188. Nº 2.}

Memoria y condisiones que seandeguardar enlaexecusion dela pintura y lenseria del Real tumulo que la Ciudad manda Hazer alas onRas del Rey nuestro señor que su declarasion es la siguiente.

- Primeramente es Condision que enlos maestros pintores enquien se Remataren ande ser obligados a poner todo el lienso que fuere menester dedar y Resebir Asiparabestir las pilastras ynteriores yexteriores encornisamentos pedrestales piramidas yReliebes y Cortinas francas y demas Remates bestidos los bastidores que les dieren enlos quales sean de pintar las figuras quepormemeoria seles dieren en los encasamentos enforma de postigos y ensima enlastargas escrebir los xeroglificos que les dieren._-

- Yes Condision que enlas Clabes delos Arcos prinsipales a la parte deafuera enlas targas que les dieren Andepintar las armas enteras de Castilla y enlas targas pequeñas Ande pintar las armas de Sevilla y enlas engutas de los arcos las palomas de la pas Conlas ramos de oliba $==\mathrm{y}$ entodo lo demas andeguardar el follage yornato que demuestra la trasa dando todo el lienso que pidiere suornato.

- Y es condision que por de dentro del primer cuerpo Adedartodo el lienso que fuere menester asi pabestir las pilastras como arcos y cornisas y para los bastidores del SeRamiento enforma de bovedas pintando enlos blancos Leones y Castillas y en los blancos sus perfiles y Romanos contodo lusimiento.

- Y es Condision queenel Cuerpo Sigundo sean de bestir de lienso todos los muros enforma de pilastras por dedentro y fuera dinteles alquitrabes Frisos y cornisas donde pidiere lienso pintando loquedemuestra la trasa yaloque fuere madera seleadedar dela color de bronce sonbreados conlos Remates deesta parte y asimismo alos frontes enla conformidad que estan enlatrasa $=\mathrm{y}$ por de dentro seade enlensar sumedia naranja en la forma que se dieren los bastidores pintados sus compartimentos = ytodos los balaustres detodo este edifisio y mesas altas y baxas selesadedar de color consus perfiles y demas Remates.

- Yes condision que la Fama que le dieren la Andeaparajar las naguas de blanco Consu bordadura de color $=$ y la cota carmesi consus faldones consusalas de colores y su banda encarnada y el Clarin ymoRion matisado.

- Yes condision que Andepintar quatro banderolas de olandilla amarilla puestas en sus Astas las armas de leones y castillas por ambas partes alas esquinas dela Cornisa alta dedos baras de Cayda puestas por su quenta $=$ y dar de color a los cañones donde entra la sera

- Yes Condision que todo lo que no se pudiere pintar dentro de la lonja loayan dehazer en el sitio donde se arma y Retocar todo lo que faltare dexandolo todo acabado entoda perfesion asatisfasion delos señores caballeros diputados y vista del maestro mayor dando de color aquatro cuerdas que lesdieren para Retenidas y siguridad del edificio = dando la mitad del dinero luego que seayan obligado y afiansado y laotra mitad como bayan obrando dandolo acabado dentro de mes y medio noquedando por su parte conmas lo que ordenare la diputasion fecho en postero de otubre de mill y seisçientos y sesenta y sinco años

Pedro ss ${ }^{\circ}$ de falconete 


\section{A.M.S. Sección V. Escribanías del siglo XVIII. Tomo 188. № 2.}

Memoria y condisiones que seandeguardar enlaexecusion del Real tumulo que la Ciudad manda Hazer Paralas onRas del Rey nuestro señor que su declarasion es como sigue.- Primera mente es condision que los maestros carpinteros que se encargaren dela execusion dicho tumulo atoda costa de Carpinteria ande obedeser latrasa en su ornato Dandole prinsipio dentro dela lonja conla tablason ymadera menuda $=$ a los quatro arcos prinsipales en sus serzas y barrotes bistiendolos de lienzo queledieren y tahuelas Ansi elgrueso como bolsores para que bayan pintando y assi en todos los bastidores delos pilastrones y entrecasos con los Reliebes de pilastras ynpostas y targas hasta el alto de Resebir la Cornisa con sus Repisas Para que se bayan pintando las figuras de los postigos y targas de ensima de los geroglifi$\cos$.

- y es Condision que en estando dispuestos los quatro pilastroses yarcos para po dello armar en la yglesia seleadedar prinsipio sobre la soleria entre los dos coros. Llevar la madera grande para haser el quadro deestribos conforme latrasa consus mortaxas para Clabar Unos conotros eyandole sus quadrantes aelterçio encalabernados enlos estribos = yaplomo de los Referidos sea de haser otro telar que empareje con la ultima grada de la entrada de la capilla mayor = y aeste peso seade en madera y entablas conpies derechos y tornapuntas sobre que ade empesar la fabrica de pilastrones y arcos = dexando suan dende bara de ancho porde fuera para paseo del Responso y sualtura consu faldamento de lienso en su bastidor pintado de canteria parda aRimando su barandal con sus mesas donde andellebar sus canones para luses que an de dar buelta ala Redonda menos en las gradas que a de aber enfrente delos quatro $\operatorname{arcos}$.

- y es condision que en los segundos pilastrones de dentro sean delebantar sobre su ymposta los quatro arcos con sus pechinas y cornisas encontorno aResebir elsielo quadrado consus bastidores enlensados y porde fuera su cornisa consus Resaltos que demuestra la traza de madera limpia = conel ornato de portadas con todas sus targas y talla para pintar las armas = pasando sus frontes por sima y entodos los blancos que ay y bestido de pilastras sea de enlensar para que se pinte enla conformidad Referida asi por defuera como por de dentro subiendo sus pinetes y arboles dende los telares de abaxo bien clavados que sirban de almas a los pilastrones baxos y altos = ysobre las cornisas de dentro sus bastidores enlensados Hasiendo su seramiento enla forma que seleordenare = ysobre el sigundo suelo enmedio seadehaser una tarima de tres gradas de alto y tres baras en quadro con la Urna que demuestra la trasa. -

- Y es condision que sobre la cornisa y fronte del ultimo cuerpo seadehaser otro telar quadrado sobre que se forma el obilisco piramidal que Remata el edificio con supinete enmedio geho fuerte que pase todo el Remate y que suba bara y media mas que la bola para donde sea de haser firme Una muger que ymite la fama bestida aeltrage que seusa desu lienso con naguas y cotas Alas ymoRion y Clarin todo pintada algo de los Pintores = yes Remata en sus tres terçios de bastidores bestidos delienzo con sus tres Ympostas de barandillas para clavar los cañones de las luces.

- Y es condision que todos los cañones Altos y baxos para luses gruesas y delgadas Andeser deo Jade lata los gruesos de quarta de peralte todos consus orejas para clabadas sobre la madera y los delgados en la mesma conformidad = pidiendo el grueso de la sera todos delgados y gruesos cortados ael talle donde ande serbir para esten a plomo. 
- yes condision que para siguridad de este edificio aya de dar a los pintores quatro cuerdas de cañamo para que les den de color que esten amarradas en los firmes de las quatro esquinas de la ultima cornisa que se hagan firmes enlos barandales delas tribunas del templo. Las dos a la parte del Coro y las dos a la capilla mayor.

- yes condision que todo lo Referido enestas condisiones loadehazer yexecutar dentro de mes y medio contodos los oficiales y gente que fuere menester sinque se haga falta a los maestros pintores asatisfasion de los cavalleros diputados yvista del maestro mayor y conforme el Remate y condisiones seadeobligar yafiansar dandole la mitad de dinero y luego la otra mitad como lo bayan obrando, yacabadas las honras y Recogido la ciudad, lo que le toca $=$ Luego sin ninguna dilasion desbarate el edificio y llebe la madera a la lonja dexando la yglesia libre ydesembarasada Reparando loque se maltratre dela soleria partiendo por yguales partes los despojos que quedaren entre los maestros carpinteros y los pintores $\mathrm{ff}^{\circ}$ enprimero de noviembre de 1665 años

Pedro Sanchez de falconete 